\title{
OS PERSAS, DE TIMÓTEO DE MiLETO: TRADUÇÃo E BREVE COMENTÁRIO MÉTRICO
}

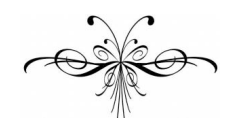

RoOSEVELT Rocha

$\mathrm{T}$ Timóteo de Mileto nasceu por volta de 450 e morreu por volta de 359 a. C. com mais ou menos 90 anos de idade. Seu nome se tornou conhecido na Antiguidade por causa de suas inovações no campo da composição poético-musical. Ele próprio se gaba, no fragmento 796 PMG (traduzido abaixo), por ter promovido inovações e por não seguir uma Musa antiquada. Segundo algumas fontes, entre elas o verso 230 do fragmento 791 PMG, de Os Persas, ele teria aumentado o número de cordas da cítara das tradicionais 7 para 10,11 ou 12 , dependendo da fonte ${ }^{1}$. Isso é um indício de que suas composições eram mais complexas, porque continham um grande número de notas musicais e utilizavam saltos intervalares menos usuais nas melodias de poetas do passado. Infelizmente, não possuímos nenhuma de suas canções para poder ter uma ideia de como a sua poesia soava quando cantada. Porém podemos ter algum vislumbre e imaginar como suas composições seriam, por exemplo, ouvindo um trecho da tragédia Orestes, (vv. 338-344 = 322-328), de Eurípides, que chegou até nós com alguma notação musical transcrita num fragmento do Papiro de Viena G 2315, publicado por Pöhlmann e West (2001, p. 18-21). Digo isso, porque algumas fontes ${ }^{2}$ falam da amizade que teria existido entre Eurípides e Timóteo. É possível que nosso poeta tenha influenciado o dramaturgo e alguma marca dessa influência possa ter ficado na melodia hoje acessível à nossa audição ${ }^{3}$.

De qualquer modo, podemos esboçar algum julgamento sobre sua obra lendo os fragmentos que chegaram até nós citados por outros autores e, principalmente, através do texto encontrado no chamado Papiro de Berlin 9875, publicado pela primeira vez por Wilamowitz, em 1903. O papiro, datado do século IV a. C. e, portanto, bastante próximo temporalmente do nosso autor, foi encontrado em 1902, em Abusir, no Egito. O texto tem 6 colunas, das quais a

\footnotetext{
${ }^{1}$ Cf. também os testemunhos 1, 2 e 7 da edição de Campbell (1993: 70-73 e 76). Ver também West, 1992: 361-364; e Csapo e Wilson, 2009: 277-293.

${ }^{2}$ Cf. test. 6, na edição de Campbell (1993: 74-77). Ver também Hordern, 2002: 4. Timóteo teria, inclusive, composto o epitáfio de Eurípides. Cf. o fragmento da Vita Euripidis em Campbell, 1993: 120-121.

${ }^{3}$ É possível ouvir essa e outras melodias da música grega antiga em gravações feitas pelo grupo Kérylos (dirigido por Annie Bélis), no disco produzido pelo De Organographia (dirigido por Philip Neumann) ou ainda no disco de Gregorio Paniagua com o Atrium Musicae de Madrid.
} 
primeira está quase ilegível, a segunda (vv. 1-59) está bastante fragmentada, mas as outras quatro últimas colunas estão relativamente bem preservadas. É possível que o começo do poema estivesse em outro rolo, que incluiria os fragmentos 788, 789 e 790. Ao todo, Os Persas devia ter por volta de 650 versos. No papiro, o texto foi escrito em forma de prosa. Wilamowitz o editou com 253 versos e Page reduziu esse número para 240. O tema central do poema é o mesmo de Os Persas, de Ésquilo, ou seja, a vitória dos gregos na batalha naval de Salamina. Mas, além disso, não possuímos maiores informações e grande parte do conteúdo do poema permanece obscuro. Timóteo o teria executado em Atenas, em 408 a. C., por ocasião de um concurso de citarodia. ${ }^{4}$ O estilo do texto é bastante complexo, como é característico de poetas da poesia lírica coral como Simônides, Píndaro e Baquílides. Timóteo, em certos momentos usa dois e algumas vezes três adjetivos para qualificar um único substantivo. Algumas vezes alguns desses adjetivos são compostos de duas e até de três raízes diferentes. Por isso, algumas vezes, na tradução, eu criei verdadeiros monstros lexicais juntando três palavras para criar uma palavra em português. Esse meu procedimento é consciente e tem como objetivo tentar reproduzir na nossa língua a estranheza que o texto de Timóteo devia causar nos seus ouvintes. Além disso, ele era também um inventor de palavras, como outros poetas gregos antigos. Por isso, encontramos no fragmento d'Os Persas alguns hapax legomenoi, palavras que só aparecem no texto de Timóteo. Ele costuma também criar metáforas de certa forma bizarras. Por exemplo, quando chama os remos de 'pés' ou 'mãos' dos barcos.

Quanto à métrica, Timóteo parece não ter sido menos inventivo. Seus primeiros nomos teriam sido compostos em hexâmetros ${ }^{5}$. Os Persas tinham um prelúdio composto nesse metro (cf. Fr. 788 PMG), feito por Eurípides, mas, no resto do poema, encontramos diferentes metros, principalmente estruturas jâmbicas mescladas a ritmos eólicos. Dionísio de Halicarnasso e Heféstion já falavam sobre a liberdade com que Timóteo compunha os seus versos ${ }^{6}$. De acordo com a análise de West (1982, p. 138-139), as linhas de 1 a 136 e 139 a 177 do papiro são predominantemente jâmbicas, com algumas ocorrências de coriambos e algumas sequências trocaicas. Algumas estruturas créticas aparecem nos versos 44-45, 116-120, docmíacas nos versos 66-69 e 75, datílicas nos versos 82 e 139, anapésticas em 88-89 e eólicas em 90-93. Entre os versos 178-201, encontramos principalmente ritmos eólicos, com algumas ocorrências jâmbicas e datílicas. No fim (202-240), Timóteo termina com estruturas eólicas, principalmente com glicôneos e ferecrácios. Desse modo, se há uma palavra que poderíamos usar para caracterizar a poesia, a música e a métrica timoteana, essa palavra é poikilía, "variedade", "colorido", "complexidade".

Quanto à tradução, posso dizer que me exigiu um certo trabalho, seja por causa do estado fragmentário do texto, seja pela dificuldade do estilo poético do autor. O texto de base é o de Page (1962), que, inclusive traz uma análise métrica um pouco diferente daquela apresentada por West, citada no

\footnotetext{
${ }^{4}$ Sobre a performance do poema, cf. Herington, 1985: 151-160.

${ }^{5}$ Cf. Plutarco, Sobre a Música, 4, 1132e, na tradução de minha autoria publicada na Coleção Autores Gregos e Latinos do Centro de Estudos Clássicos e Humanísticos da Universidade de Coimbra, disponível na página https://bdigital.sib.uc.pt/jspui/handle/123456789/43.

${ }^{6}$ Cf. os testemunhos 10 e 11, da edição de Campbell (1993: 78-91).
} 
parágrafo anterior. Porém, segui muitas sugestões de Hordern (2002), por causa da erudição do seu comentário e da grande competência demonstrada no que que diz respeito à papirologia. Esse autor apresenta também suas próprias interpretações acerca da métrica (Hordern: 2002, p. 55-60). Porém, mesmo que haja divergências entre os intérpretes, o quadro final é sempre o mesmo: Timóteo foi um inventor radical seja com as palavras, seja nas melodias, seja no uso dos metros.

\section{Traduções}

Fr. 788 PMG = Plutarco, Vida de Filopêmen, 11 (Hexâmetro)

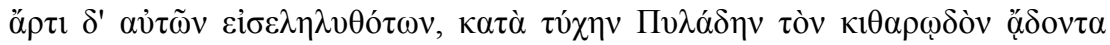

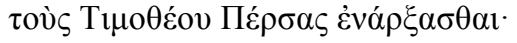

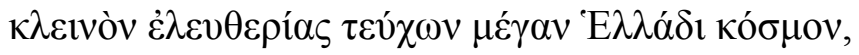

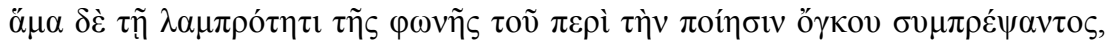

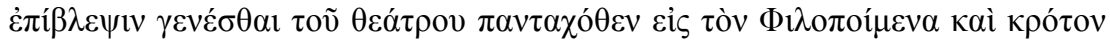

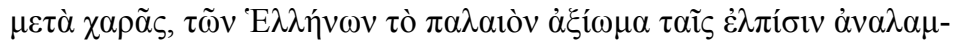

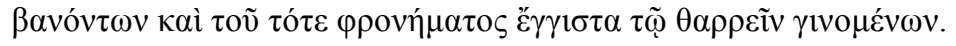

Logo que entraram [Filopêmen e suas tropas em visita ao teatro de Nemeia, em 207 a. C.], sucedeu que o citaredo Pilades estava executando Os Persas, de Timóteo, e começou:

criando $^{7}$ um ínclito e grande ornamento de liberdade para a Hélade.

e graças à esplêndida voz do cantor e da igualmente conspícua majestade da poesia, todos os expectadores voltaram seus olhos para Filopemen, e os gregos irromperam em aplausos, pois tinham a esperança de que eles estavam recuperando seu antigo prestígio e tinham a confiança de estar se reaproximando do espírito do passado.

Fr. $789 \mathrm{PMG}=$ Plutarco, De audiendis poetis, 11.

(Estrutura semelhante a um Trímetro Jâmbico com resoluções e escazonte no primeiro pé)

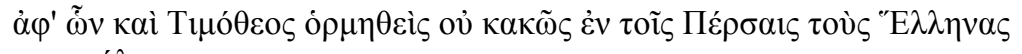
$\pi \alpha \rho \varepsilon \kappa \alpha ́ \lambda \varepsilon 1$

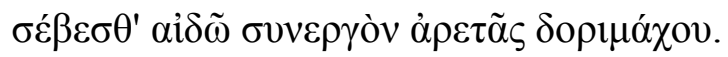

\footnotetext{
${ }^{7}$ Referindo-se talvez a Temístocles ou ao povo de Atenas. Cf. Hordern (2002: 128-129), que defende a segunda hipótese.
} 
Timóteo baseou-se nestas linhas [Ilíada, 16, 422 e 13, 121 ss.] para compor a esplêndida exortação aos gregos no seu Os Persas:

venerai o respeito ajudante da virtude lanciguerreira

Fr. 790 PMG = Plutarco, Vida de Agesilau, 14.4 (Trímetro Jâmbico seguido de uma sílaba breve)

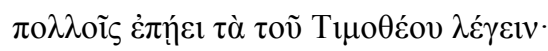

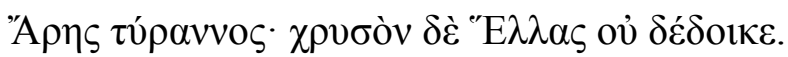

muitos [dos gregos da Ásia Menor] foram levados a citar as palavras de Timóteo:

Ares tirano: A Hélade não teme o ouro

ou

Ares é nosso senhor

ou

A Guerra é nossa senhora

Fr. 790a = Fr. 1027f PMG = Dionísio de Halicarnasso, De Compositione

Verborum, 15, 110 (Estrutura com cinco créticos)

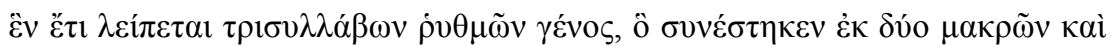

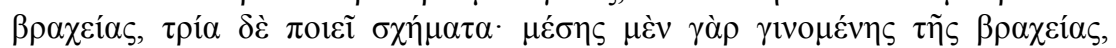

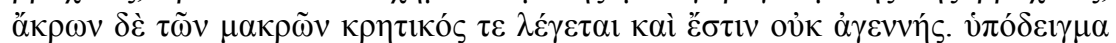

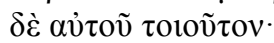

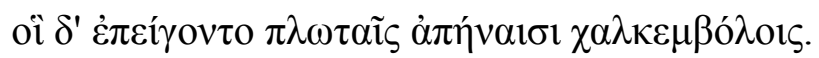

resta ainda um gênero de ritmos trissilábicos, que é composto de duas longas e uma breve. Ela assume três formas. Quando a breve está no meio e as longas nas extremidades, ele é chamado de 'crético' e não falta nobreza. Um exemplo desse tipo de ritmo é:

e eles se apressaram com seus carros flutuantes de brônzeos bicos

Fr. 791 PMG (Papyrus Berolinensis 9875)

fr. $4.3 v v \mu \varphi \alpha .[], v \alpha 1 \alpha v \pi[, 4 \varepsilon \pi[\varepsilon] v \kappa v \kappa \lambda[$,

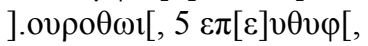

]. $\sigma \kappa о \pi \varepsilon \imath v[, 6 \sigma v[v] \delta \rho \circ \mu[, 12] \pi \alpha \lambda \mu \mu \mu[, 13$ ]$\mu о v \sigma \alpha[$

fr. 3.3$] \sigma \tau \mathrm{o} \chi \mathrm{o}[, 11] \pi \mathrm{\rho} \rho \mathrm{o}[$, fr. 7.3$] \pi \mathrm{\iota} \varphi \mathrm{o} \beta \alpha[, 4$ ] $\varepsilon 0 \xi \mathrm{v}[$

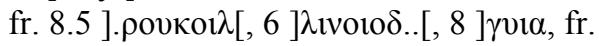
$9.6] .1 \alpha \delta 10 \pi \lambda[, 9$ col. i

fr. 1.3 ninfa (?)

fr. 1.4 sobre (?) bem arredondado

fr. 1.5 direto

fr. 4.11 de novo

fr. 3.3 fileiras (Wilamowitz) / contra as laterais (Diggle) 
$\operatorname{col1.5}] \alpha \sigma \varepsilon \imath \chi 0 v[$

$\operatorname{col} 2.1$

[..].[..].[.....] $\alpha v \tau[\quad] v \omega v[\quad] .$. $\sigma v v[\varepsilon \mu] \beta \mathrm{o} \lambda \mathrm{o}[\mathrm{l}] \sigma \mathrm{l}$

$\gamma \varepsilon 1 \tau[\ldots] \sigma[.] v.[\ldots \ldots . ..] \alpha v \tau \imath \alpha 1[\ldots \ldots ..] \pi \rho_{1}[. .]. v \varepsilon$ $\chi \alpha \rho \alpha[\xi] \alpha v \pi 0.1 \delta \varepsilon \gamma \varepsilon[\ldots] \lambda \mathrm{\gamma} \gamma \chi \mathrm{o}[\ldots \ldots ..] \alpha \mu \varphi \varepsilon \theta[\varepsilon] \nu \tau$

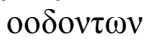

$\sigma \tau о .[.] . \alpha 1 \delta[.] \kappa v \rho \tau o 1[\sigma 1] \kappa \rho \alpha \sigma \mathrm{v} v[\ldots \ldots .$.

[ ] ]

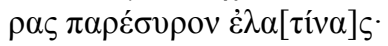

$\dot{\alpha} \lambda \lambda^{\prime} \varepsilon \dot{\imath} \mu \grave{\varepsilon} v[\hat{\varepsilon}] v \theta \dot{\varepsilon} v \delta \varepsilon[\ldots . .] .1 \varsigma-$

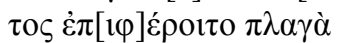

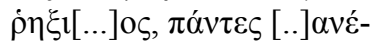

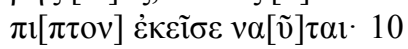

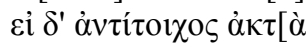

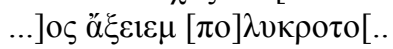

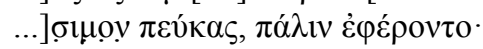

$\alpha i \delta \varepsilon[\ldots ..] \alpha \imath . \eta \gamma v \tilde{i} \alpha[\delta] \operatorname{la\varphi } \varepsilon \operatorname{cov} \alpha[1$

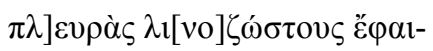

vov, $\tau \alpha \varsigma .[\ldots ..] \cdot[. ..] \ldots[.] .1 \varsigma$

$\sigma \kappa \eta \pi \tau[..] \dot{\varepsilon} \pi \varepsilon \mu \beta \alpha \dot{\alpha} \lambda \lambda[0] v \tau \varepsilon \varsigma \dot{\alpha} v \varepsilon-$

$[\chi] \alpha i ́ \tau 1 \zeta o v, \alpha i \delta \varepsilon \dot{~} \pi \rho \alpha[v \varepsilon ́ \varepsilon \varsigma$

......].[...] $\alpha \varsigma \dot{\alpha} \pi \eta \gamma<\lambda>\alpha \ddot{-}-$

$\sigma \mu \varepsilon ́ v \alpha[1] \sigma \imath \delta \alpha[\rho]<\varepsilon<>1 \kappa \rho \alpha ́ v \varepsilon \imath$.

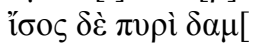

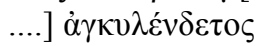

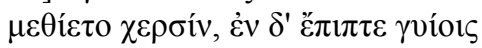

$\alpha i \theta \varepsilon[\ldots . . . . ..] \omega \dot{\omega} \mu \alpha \grave{\alpha} \delta \alpha \kappa \rho \alpha \delta \alpha i ́ v \omega v$.

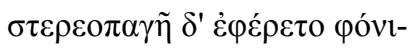

$\alpha[\ldots \ldots . . ..] \alpha[.$.$] ... \tau$ $\tau \varepsilon \pi \varepsilon \rho i ́ \beta o \lambda \alpha$

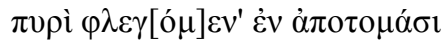

$\beta o v \delta o ́[\rho o 1 \sigma \cdot \cdot \tau \tilde{\omega} v \delta \grave{\varepsilon}] \beta i ́ o \tau o \varsigma$

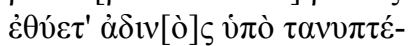

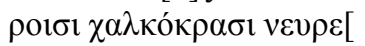

$\sigma \mu \alpha \rho \alpha \gamma \delta o \chi \alpha i ́ \tau \alpha \varsigma \delta \grave{\varepsilon} \pi o ́ v-$

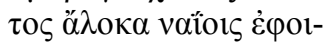

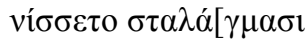

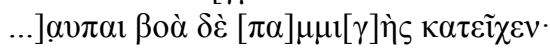

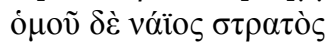

$\beta \alpha ́ \rho \beta \alpha \rho \varsigma \dot{\alpha} \mu \mu 1[\gamma \ldots . .$.

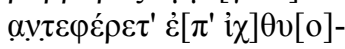

$\sigma \tau \varepsilon \dot{\varphi} \varphi \sigma 1 \mu \alpha \rho \mu \alpha \rho \circ \pi[\tau u ́ \chi] \mathrm{o}[1] \varsigma$

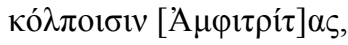

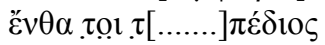

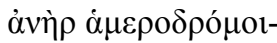

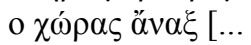

....] $\mu \beta \rho i ́ \alpha v \alpha . \omega[\ldots$

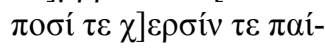

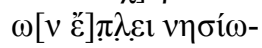

fr. 6.2 tumultuará (?)
fr. 8.6 de linho

fr. 8.8 membros

col. ii-vi

...com os esporões vizinhos(?)...

...sulcaram com os pés...lança(?)...

...postos em torno dos dentes os gemidos...

mas eles com as cabeças curvadas

... as mã-

os arrancaram de abeto;

mas se de um lad[o irresis]tí-

vel (?) lançassem golpe

romperremo, todos juntos ca-

íriam para trás ali os nautas;

e se um promontório do outro lado

quebrasse [...] os navegantes

pinhos de muitos bancos, ${ }^{8}$ de novo eles cairiam.

e quando umas naves arrancando os impotentes

membros

os flancos linho-amarrados reve- 15

laram, de outras naves, com plúmbeos

raios caindo em cima vi-

raram, outras naves invertidas

[afundaram] de seus ornamentos pri-

vadas pelo elmo de ferro. ${ }^{9}$

Igual ao fogo o [dardo]doma-

[homem] amarrado com correira

foi arremessado pelas suas mãos e caiu entre os membros

arcorredor, ${ }^{10}$ sacudindo seus corpos com violência.

E os soliduros assassinos eram lança-

25

dos [dardos?] e os tecidos

ardendo em fogo nas flechas

boitrespassantes. E a vida pelos dardos

longialados bronzepontudos cordi[rretesados]

estava sendo sacrificada em grande número. 30

E o esmeraldacomado mar

em seus campos purpurejou

com os navais [inimigos],

$\mathrm{E}$ ao [gr]ito comesclado o clamor prevalecia.

E em conjunto a frota naval

bárbara em con[fusão]

foi rechaçada para os peixe-

entrelaçados brilhantedrapejados

regaços de [Anfitrite].

Então um homem da [...]

planície, senhor

da terra percorrível em um dia [...

golpeando] a água

com seus pés e mãos

nadou, um insular,

\footnotetext{
${ }^{8}$ Ou seja, os remos.

${ }^{9}$ Os esporões.

${ }^{10}$ Referindo-se ao dardo.
} 
$\tau \alpha \varsigma[\ldots . . ..] \varsigma$ @ę̣ivó $\mu \varepsilon[v o \varsigma$

$\ldots \delta] 1 \varepsilon \xi o ́ \delta o v \varsigma \mu[\alpha \tau \varepsilon v ́ \omega] v$

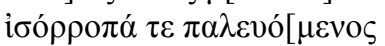

[ $] \eta \lambda[] \omega v$

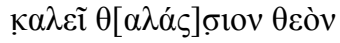

$\pi \alpha ́ \tau \varepsilon \rho \alpha \tau[$

.......].vo[...] $\varphi 1[] . \kappa \varepsilon \pi[..] . .[\ldots \ldots \ldots . ..] \lambda \alpha \sigma \sigma \omega v .[$

.......] $\sigma \pi[..] \tau \varepsilon[] . . \gamma \alpha v[..] \mathrm{\rho}[\ldots . . . . . . . ..] \alpha \pi \varepsilon \rho \sigma \alpha \nu$

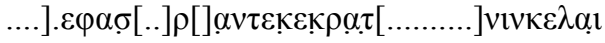

$\ldots \alpha \mu] \beta \lambda v \delta \omega[\chi] \rho \circ v[] \sigma \kappa \alpha \tau \varepsilon \sigma \sigma \varphi \rho \alpha[\ldots \ldots.] . \sigma \tau \alpha \quad 55$

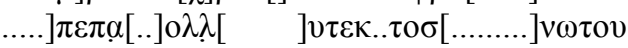

.....] $\varepsilon \delta 1 \alpha \pi \alpha \lambda \varepsilon v \omega v[$ [ ]

.....] $\varepsilon \sigma \mu[\mathrm{o}] \varsigma[\alpha \pi] \varepsilon 1 \rho \circ \varsigma[] \varphi \mathrm{iv} \alpha \mathrm{ot} \sigma \tau \rho v[\ldots . ..] \lambda \mathrm{\imath} \chi \theta \varepsilon 1 \varsigma$

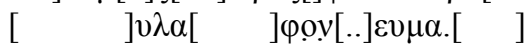

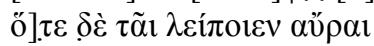

$\tau \tilde{\alpha} \imath \delta^{\prime} \dot{\varepsilon} \pi \varepsilon 1 \sigma \varepsilon ́ \pi \imath \pi \mathrm{ov}, \dot{\alpha} \varphi \rho \tilde{\varphi} \imath$

$\delta^{\prime}<\tilde{v}^{\prime}>\dot{\alpha} \beta \alpha \kappa \chi i ́ \omega \tau$ os ő $\mu-$

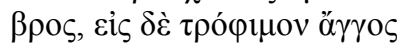

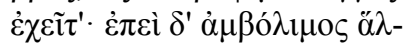

$\mu \alpha \sigma \tau o ́ \mu \alpha \tau o \varsigma$ vं $\pi \varepsilon \rho \varepsilon \dot{\theta} \theta 1 \varepsilon v$,

ỏ $\xi v \pi \alpha \rho \alpha v \delta \eta ́ \tau \omega 1$

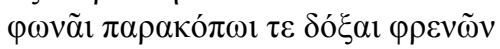

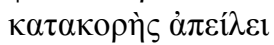

$\gamma o ́ \mu \varphi 01 \varsigma<1 v>\dot{\varepsilon} \mu \pi \rho i ́ \omega v$

$\dagger \mu \mu \mathrm{ov} \mu \varepsilon v o \varsigma \dagger \lambda v \mu \varepsilon \tilde{\omega}-$

vi $\sigma \omega ́ \mu \alpha \tau o \varsigma \theta \alpha \lambda \alpha ́ \sigma \sigma \alpha l$.

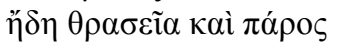

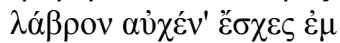

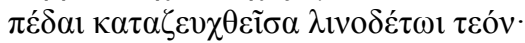

vข̃v $\delta \varepsilon \dot{\varepsilon} \sigma^{\prime} \dot{\alpha} v \alpha \tau \alpha \rho \alpha ́ \xi \varepsilon 1$

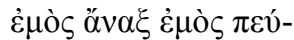

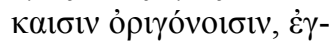

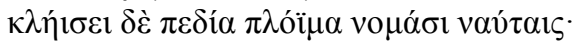

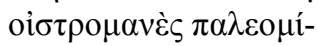

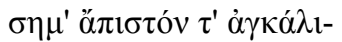

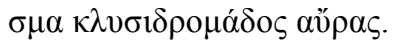

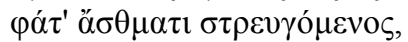

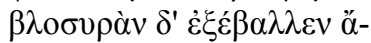

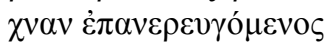

$\sigma \tau o ́ \mu \alpha \tau \imath \beta \rho v ́ \chi 10 v$ ő $\lambda \mu \alpha v$.

85

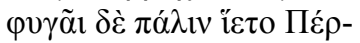

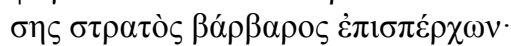

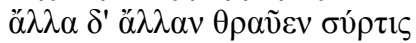

$\mu \alpha \kappa \rho \alpha v \chi \varepsilon v o ́-$

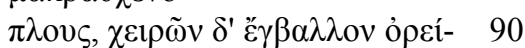

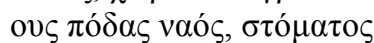

$\delta^{\prime} \dot{\varepsilon} \xi \dot{\eta} \lambda \lambda o v \tau о \mu \alpha \rho \mu \alpha \rho \circ \varphi \varepsilon \gamma-$

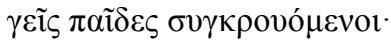

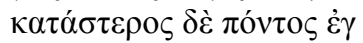

$\lambda ı$ เ $\pi v o ́ \eta \varsigma . . .[.] \sigma \tau \varepsilon \rho \varepsilon ́ \sigma ı$

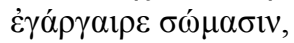

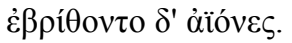

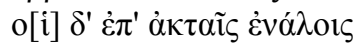

\footnotetext{
${ }^{11}$ Ou seja, estômago.

12 Os remos.

${ }^{13}$ Os dentes.
}

sendo arremessado (?)

buscando meios de escapar

e caindo na rede $[. .$.

igualmente equilibrado..

Ele chama o [marinho?] deus

e o pai...

$\cdots$

...Persas...

...(negro?)...

...brusco, pálido...

...acessível saída...

...prisão infinita...naval..

...agarrados a destroços navais (?)...

E ora com o vento eles partiam

ora atacavam, mas com espuma

caia a abáquica chu-

va e para o seu vaso alimentar ${ }^{11}$

escorria. E quando a ressurgente sal-

moura da boca enfureceu-se,

com agudinumana

voz e com pensamento frenético no espírito, sem moderação desafia

rangendo seus dentes

enraivecido com o mar,

o destruidor do seu corpo:

"Mesmo antes de agora, arrogante,

teu turbulento pescoço tiveste numa

peia linhitrançada, tu cojungido.

E agora te agitará

o meu senhor, o meu, com seus pi-

nhos montigerados e

encerrará tuas planícies navegáveis com seus nômades

nautas,

tu enfurecido antigo ob-

jeto de ódio e pérfido predi-

leto do velociumectante vento."

Disse num fôlego sofrendo

e cuspiu uma horrenda

espuma vomitando

com a boca a profunda salmoura. 85

E em fuga recuou do Per-

sa o exército bárbaro, apressando-se.

E uma linha de navios despedaçou a outra

que navegava o grande pescoço

(do mar) e de suas mãos lançaram os mon- 90

teses pés da nave, ${ }^{12} \mathrm{e}$ da boca

saltaram seus alvibrilhan-

tes filhos ${ }^{13}$ golpeados juntos.

E o estrelado mar en-

xameado com corpos

desprovidos de luz pela falta de ar

e as praias estavam carregados.

E outros, nas escarpas à beira-mar 


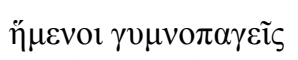

$\alpha \tau \tau \tilde{\alpha} \imath \tau \varepsilon \kappa \alpha \grave{~} \delta \alpha \kappa \rho v-$

$\sigma \tau \alpha \gamma \varepsilon \tilde{\imath}[\gamma]$ ó $\omega$ ı

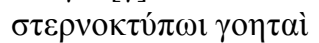

$\theta \rho \eta v \omega ́ \delta \varepsilon \imath ~ \kappa \alpha \tau \varepsilon i ́ \chi o v \tau^{\prime}$ ỏ $\delta \rho \mu \tilde{\omega} \iota$.

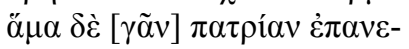

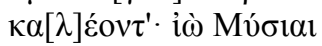

$\delta \varepsilon v \delta \rho \circ \varepsilon \dot{\theta \varepsilon \imath \rho \alpha} \pi \tau v \chi \alpha i$,

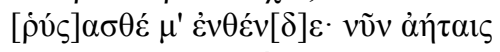

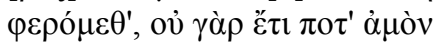

$[\sigma \tilde{\omega}] \mu \alpha \delta \varepsilon \dot{\xi} \xi \tau \alpha \imath[\pi \delta ́ \lambda] ı$

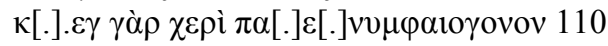

[...].ov $\alpha v \tau \rho o v o[\ldots] .[\ldots] \delta 1 \alpha \sigma \tau \alpha \kappa \alpha \pi \varepsilon[\ldots . .$.$] .$

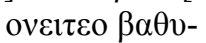

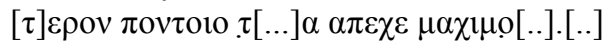

$\pi \lambda$ ouov E $\lambda \lambda \alpha \nu \varepsilon v[\ldots] \eta$

$\sigma \tau \dot{\varepsilon} \gamma \eta \nu$ है $\delta \varepsilon \mu \varepsilon$

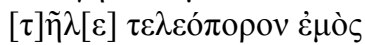

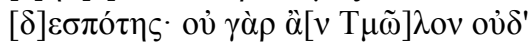

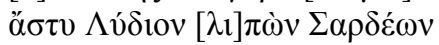

$\tilde{\eta} \lambda \theta 0 v\left[{ }^{\circ} E\right] \lambda \lambda \alpha v^{\prime} \dot{\alpha} \pi \varepsilon \dot{\varepsilon} \rho \xi \omega$ 'A $\rho[\eta$.

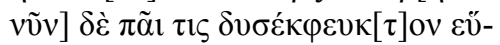

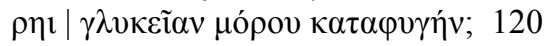

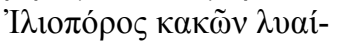

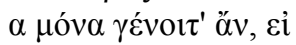

$\dagger \delta v v \alpha \sigma \tau \alpha \dagger \pi \rho{ }^{\prime} \varsigma \mu \varepsilon \lambda \alpha \mu-$

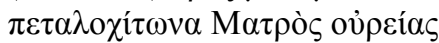

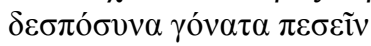

$\varepsilon \dot{v} \omega \lambda \varepsilon \dot{\varepsilon} v 0{ }^{\prime} \tau \varepsilon \chi \varepsilon \bar{i} \rho \alpha \varsigma \dot{\alpha} \mu \varphi 1 \beta \alpha \dot{\alpha} \lambda \lambda \omega v$

$\dagger \lambda \operatorname{li} \sigma \sigma \omega v \dagger \propto \rho v \sigma o \pi \lambda \operatorname{có}_{\alpha} \alpha \mu \varepsilon$

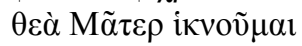

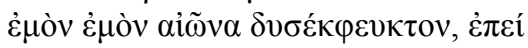

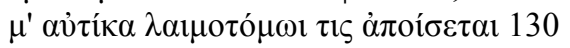

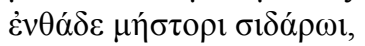

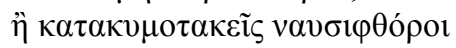

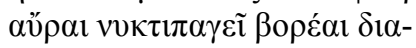

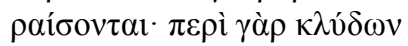

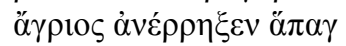

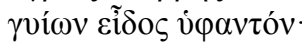

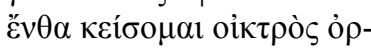

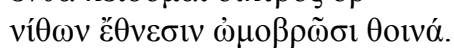

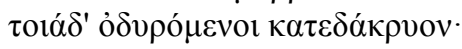

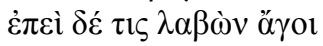

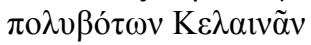

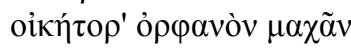

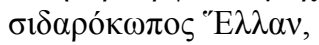

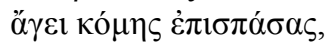

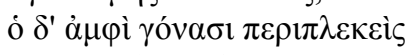

$\dot{\varepsilon} \lambda \hat{i} \sigma \sigma \varepsilon \tau^{\prime},{ }^{\prime} E \lambda \lambda \alpha \alpha^{\prime}{ }^{\prime} \dot{\varepsilon} \mu \pi \lambda \varepsilon \dot{\varepsilon} \kappa \omega v$

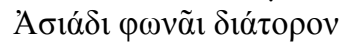

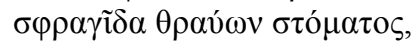

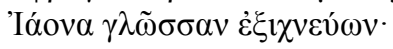

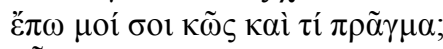

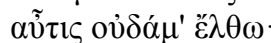

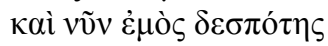

sentados nudigelados

com grito e com destila-

100

lágrima lamento

golpeiapeito os chorosos

derramavam trenódico pranto.

Juntos a terra pátria evo-

cavam: "Ió, Mísios

105

arboreocomados vales,

resgatai-me daqui. Agora por rajadas

somos levados, pois nunca mais a minha

cidade receberá o meu corpo

...pois com a mão...o há muito tempo (?) ninfeugera-

do

impenetráv]el antro..

$110 \mathrm{a}$

...o mais profundo abismo do mar...

Afasta o combatente

naval heleno (?)...

[Quem dera] não tivesse construído a cobertura

que fez o cruzamento o meu

115

mestre, pois nem o Tmolo nem

a cidade Lídia de Sardes tendo deixado

eu não viria afastar o Ares heleno.

Mas agora onde alguém encontrará um doce

refúgio contra a morte dura de evitar?

A passagem de Ílion seria

a única libertadora dos males, se

é possível diante dos joelhos

senhoris escurifolhivestidos

da montanha da Mãe cair

e lançando em torno as belas mãos suplicaria: 'Salva, auritrançada

deusa Mãe, peço-te,

a minha, minha vida dura de escapar, já que

alguém logo me levará embora daqui

com o cortagarganta inspirador ferro,

ou os sublevaondas arruinanaves

ventos com um noctirregelante Bóreas des-

pedaçar-me-ão, pois em torno a onda

selvagem arrebentou toda

a forma bem-trançada dos membros.

Aqui jazerei, miserável, ban-

quete para as tribos carnívoras das aves."

Tais coisas pranteando vertiam lágrimas.

Mas sempre que um heleno ferriarmado 140

prendia e levava

da multinutriz Celênia

um habitante órfão das batalhas,

levava-o arrastando-o pela coma,

e ele ${ }^{14}$ abraçando seus joelhos

suplicava, entrançando à helena

uma voz asiática o perfurado

selo fraturando da boca,

da língua jônica seguindo o rastro:

"Como mim falar você e que coisa?

Mais nunca venho.

Mesmo agora meu senhor

\footnotetext{
${ }^{14} \mathrm{O}$ celênio.
} 


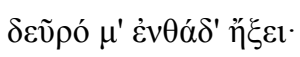

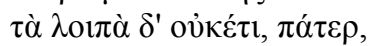

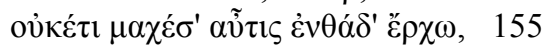

$\dot{\alpha} \lambda \lambda \dot{\alpha} \kappa \alpha ́ \theta \omega$.

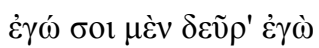

$\kappa \varepsilon i ̃ \sigma \varepsilon \pi \alpha \rho \grave{\alpha} \Sigma \alpha ́ \rho \delta 1, \pi \alpha \rho \grave{\alpha} \Sigma o v ̃ \sigma \alpha$,

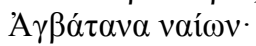

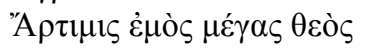

$\pi \alpha \rho^{\prime}$ 'Е $\varphi \varepsilon \sigma o v$ $\varphi v \lambda \alpha \dot{\xi} \varepsilon 1$.

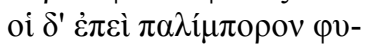

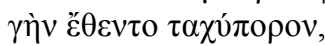

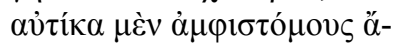

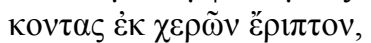

$\delta \rho v ́ \pi \tau \varepsilon \tau \mathrm{o} \delta \dot{\varepsilon} \pi \rho \sigma^{\circ} \sigma \omega \pi^{\prime}$ o้vv$\xi_{1}$.

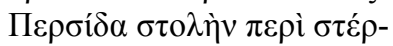

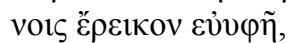

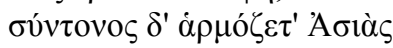

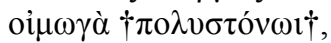

$\kappa \tau v \pi \varepsilon \tilde{\imath} \delta \dot{\varepsilon} \pi \tilde{\alpha} \sigma \alpha \mathrm{B} \alpha \sigma \imath \lambda \varepsilon \dot{\varepsilon} \omega \varsigma \pi \alpha v \eta ́ \gamma v \rho \imath \varsigma$

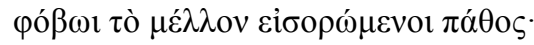

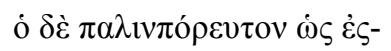

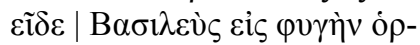

$\mu \tilde{\omega} \nu \tau \alpha \pi \alpha \mu \mu \imath \gamma \tilde{\eta} \sigma \tau \rho \alpha \tau o ́ v$,

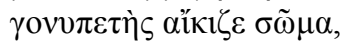

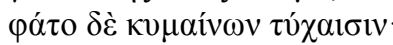

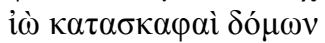

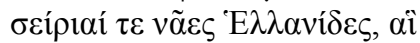

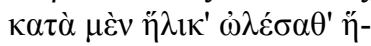

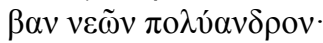

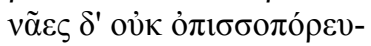

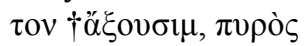

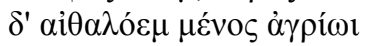

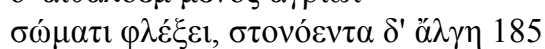

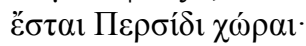

ì̀ $\beta \alpha \rho \varepsilon \tilde{\imath} \alpha$ $\sigma v \mu \varphi о \rho \alpha ́$,

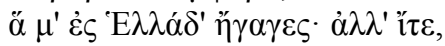
$\mu \eta \kappa \varepsilon ́ \tau 1 ~ \mu \varepsilon ́ \lambda \lambda \varepsilon \tau \varepsilon$,

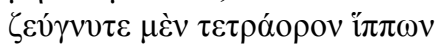

ő $\chi \eta \mu$ ', oi $\delta^{\prime} \alpha \dot{\alpha} v \alpha ́ \rho 1 \theta \mu$ ov ő $\lambda$ -

ßov

$\pi \dot{\mu} \mu \pi \rho \alpha \tau \varepsilon \delta \dot{\varepsilon} \sigma \kappa \eta v \alpha ́ \varsigma$,

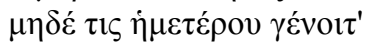

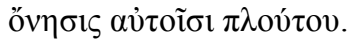

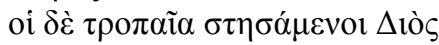

$\dot{\alpha} \gamma \nu \tau_{\tau} \alpha \tau \sigma \nu \tau \varepsilon \dot{\mu} \mu \varepsilon v o \varsigma, \Pi \alpha 1 \tilde{\alpha} v^{\prime}$

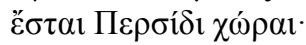

ő้ $\alpha \kappa \tau \alpha, \sigma o ́ \mu \mu \varepsilon \tau \rho \circ 1 \delta^{\prime} \dot{\varepsilon} \pi \varepsilon-$

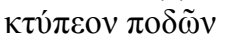

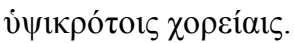

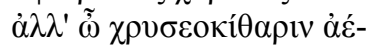

$\xi \omega \nu \mu о \tilde{v} \sigma \alpha \nu v \varepsilon 0 \tau \varepsilon v \chi \tilde{\eta}$,

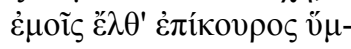

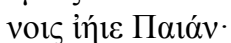

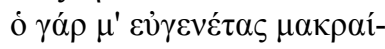

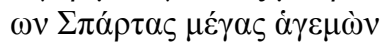

$\beta \rho u ́ \omega v$ ơv $\theta \varepsilon \sigma \mathrm{iv} \eta ๊ \beta \alpha \varsigma$

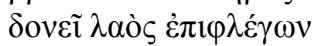

aqui para cá me trouxe.

No futuro nunca mais, meu pai,

nunca mais para lutar de novo para cá venho 155

mas fico.

Eu contigo não aqui, eu

ficarei em Sardis, em Sussa,

morando em Agbatana.

Ártimis, meu grande deus,

em Éfeso me guardará."

E quando eles terminaram

seu recuo em fuga acelerada,

logo arremessaram de suas mãos

suas setas de dupla boca,

e seus rostos foram arranhados pelos pregos.

Sua bem-tecida veste persa

rasgaram em torno ao peito,

e altissonante lamento da Ásia

foi entoado com grande tristeza (?),

e ressoou todo o séquito do Rei

com medo olhando para o sofrimento que estava por vir.

E quando o Rei viu

o exército todoconfuso movendo-se

para trás em fuga,

caindo de joelhos maltratou seu corpo,

e disse agitando-se com sua má sorte:

"Ió, ruínas da minha casa,

e incendiárias naves helênicas, que

destruístes a numerosa idade

juvenil dos meus jovens,

as naves não os levarão

na viagem de volta, mas do fogo

a ardente força os queimará

com seu selvagem corpo e doloroso sofrimento 185

haverá na terra persa.

Ió, opressivo destino,

que me trouxeste à Hélade. Mas ide,

não mais vos demoreis,

jungi minha carruagem de quatro

cavalos, e vós carregai meu

incontável tesouro para as carroças.

Queimai as tendas,

e nenhuma vantagem haja

para eles da nossa riqueza."

E os outros colocaram troféus como um templo sacríssimo de Zeus e o Peã invocaram, o senhor

que cura, e no mesmo ritmo ba-

teram dos seus pés

com danças de alto estrépito.

Mas ó tu que exaltas a auri-

citárica Musa recém-criada,

vem como aliado dos meus hi-

nos, ié Peã que cura.

Pois o bem-nascido antigo

grande soberano de Esparta,

o povo florescente com as flores

da juventude, agita-me inflamando-se 


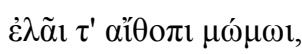

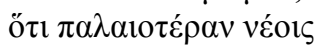

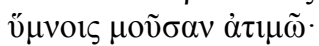

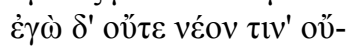

$\tau \varepsilon \gamma \varepsilon \rho \alpha o ̀ v$ oủ $\tau^{\prime}$ iøń $\beta \alpha \nu$

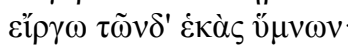

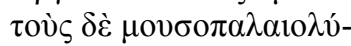

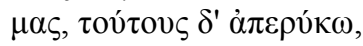

$\lambda \omega \beta \eta \tau \tilde{\eta} \rho \alpha \varsigma \dot{\alpha} 01 \delta \tilde{\alpha} v$,

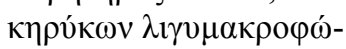

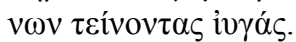

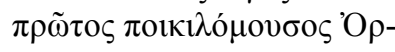

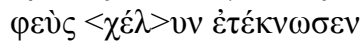

viò $\varsigma \alpha \lambda \lambda$ ió $\pi \alpha<\varsigma$

- $>>1 \varepsilon \rho i ́ \alpha \theta \varepsilon v$.

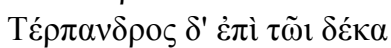

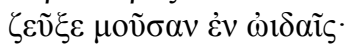

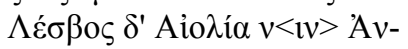

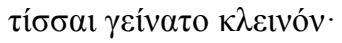

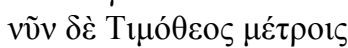

$\dot{\rho} v \theta \mu$ оi் $\tau^{\prime} \dot{\varepsilon} v \delta \varepsilon \kappa \alpha \kappa \rho о v \mu \alpha ́ \tau o เ \varsigma$

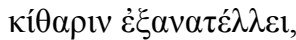

$\theta \eta \sigma \alpha v \rho o ̀ v ~ \pi o \lambda v ́ v \mu v o v$ oü-

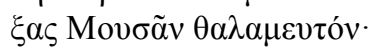

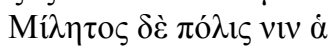

$\theta \rho \varepsilon ́ \psi \alpha \sigma^{\prime} \dot{\alpha} \mid \delta v \omega \delta \varepsilon \kappa \alpha \tau \varepsilon \imath \chi \dot{\varepsilon} о \varsigma$

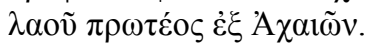

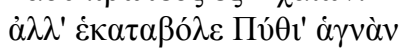

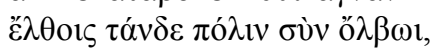
$\pi \dot{\varepsilon} \mu \pi \omega \nu \dot{\alpha} \pi \dot{\eta} \mu \mathrm{ov \imath} \lambda \alpha \tilde{\omega} 1$

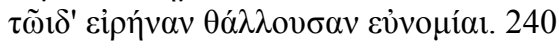

e me persegue com flamejante reprovação, 210

porque desonro a Musa

mais antiga com meus novos hinos.

Mas eu nem um jovem nem

um velho nem um coetâneo

mantenho distante destes hinos.

Mas os musantiquadoscorrupto-

res, esses eu afasto,

destruidores de cantos,

que estendem os gritos dos

arautos agudaltivóceos.

Primeiro o musintrincado Or-

feu a [quelô]nia ${ }^{15}$ engendrou,

filho de Calíope,

...da Piéria.

Terpandro, depois dele, jungiu

a Musa com dez canções. ${ }^{16}$

A eólia Lesbos em An-

tissa gerou-o, ínclito.

Agora Timóteo com metros

e ritmos onzicórdios

a cítara faz renascer,

depois de abrir o tesouro multínico

talamoguardado das Musas.

Mileto é que o nutriu,

a doze vezes murada cidade

do povo primeiro entre os Aqueus.

Ó longicerteiro Pítio, vem

a esta sacra cidade com prosperidade, enviando a este povo incólume

a paz florescente com a boa ordem.

\section{Fr. 796 PMG = Ateneu, Banquete dos Sofistas, 3. 122cd (jônico a minore)}

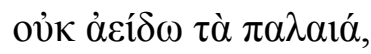
$\kappa \alpha v \alpha \grave{~ \gamma \alpha ̀ \rho ~ \alpha ் \mu ~}$

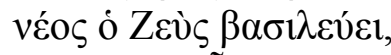

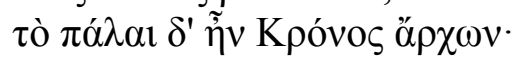

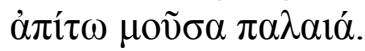

não canto velharias, minhas novidades são melhores;

um novo Zeus reina,

no passado Crono governou:

vá de retro, Musa velha!

ou

\footnotetext{
${ }^{15}$ Quelônia refere-se à lira cuja caixa de ressonância era feita com a carapaça de uma tartaruga.

${ }^{16}$ Referência às dez cordas da lira, número que teria sido estabelecido por Terpandro, segundo Timóteo.
} 
não canto velhas canções, as minhas novas são melhores; um novo Zeus reina, no passado Crono era arconte: Que afaste-se a velha Musa.

Fr. 802 PMG = Plutarco, De laude ipsius, 1, 539c

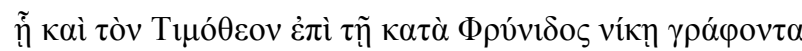

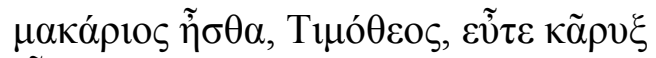

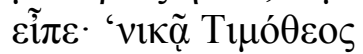

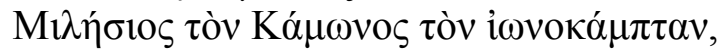

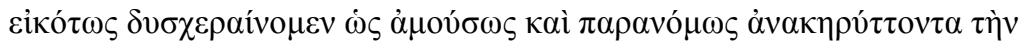

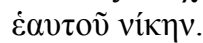

Então quando Timóteo escreve sobre sua vitória sobre Frínis,

Venturoso foste, Timóteo, quando o arauto disse: "vence Timóteo Milésio o filho de Cámon, o jonicomodulador".

temos boas razões para desaprovar a sua falta de tato e sua maneira ofensiva de se gabar por sua vitória.

Roosevelt Rocha rooseveltrocha@yahoo.com.br Universidade Federal do Paraná 


\section{Referências bibliográficas}

CampBell, David A. (1993) Greek Lyric. Vol. V: The New School of Poetry and Anonymous Songs and Hymns. Cambridge (MA) and London: Harvard University Press (Loeb Classical Library vol. 144).

CsAPO, Eric e Wilson, Peter (2009) 'Timotheus the New Musician', em Budelmann, Felix (ed.) The Cambridge Companion to Greek Lyric. Cambridge: Cambridge University Press, pp. 277-293.

Herington, John (1985) Poetry into Drama. Berkeley: University of California Press.

Hordern, James H. (2002) The Fragments of Timotheus of Miletus. Oxford: Oxford University Press.

Page, Denys (1962) Poetae Melici Graeci. Oxford: Clarendon.

Pöhlmann, Egert e West, Martin L. (2001) Documents of Ancient Greek Music. Oxford: Oxford University Press.

Plutarco (2010) Sobre o Afecto aos Filhos e Sobre a Música. Tradução do Grego, Introdução e Notas de Carmen Soares e Roosevelt Rocha. Coimbra: Centro de Estudos Clássicos e Humanísticos da Universidade de Coimbra.

West, Martin L. (1982) Greek Metre. Oxford: Clarendon.

—. (1992) Ancient Greek Music. Oxford: Clarendon. 